\title{
Pulmonary hypertension associated with congenital cardiac disease
}

\author{
Rolf M.F. Berger \\ Center for Congenital Heart Diseases, University Medical Center Groningen, University of Groningen, \\ The Netherlands
}

Keywords: Eisenmenger syndrome; systemic-to-pulmonary shunts; advanced therapies; clinical trial; evidence-based

"A wise man proportions his belief to the evidence...."

(D. Hume, 1748)

\section{Pulmonary arterial hypertension}

$\mathrm{P}$ ULMONARY ARTERIAL HYPERTENSION IS A RARE, progressive pulmonary vascular disease with an estimated prevalence of 15 to 40 per million persons. ${ }^{1}$ The disease may be idiopathic or associated with a variety of underlying conditions, including collagen vascular diseases, hepatic disease, disease produced by the human immunodeficiency virus, or congenital cardiac diseases. Over the last decade, great progress has been achieved in the understanding, the management and the prognosis of this orphan disease. The general acceptance of a clinical classification for pulmonary hypertension, as proposed and modified at subsequent world congresses in Evian, Venice and, recently, Dana Point, has allowed a certain level of standardization in diagnosis and treatment, and has made it possible to conduct clinical trials in relatively homogeneous and well-characterized populations. ${ }^{2}$ In fact, it is impressive to note that, in an orphan disease such as pulmonary arterial hypertension, 23 randomized controlled trials have been reported the last 12 years. ${ }^{3}$ Despite certain limitations of these trials, including the lack of controlled long term studies

Correspondence to: Prof dr R.M.F. Berger, Center for Congenital Heart Diseases, Dept of Paediatric Cardiology, Beatrix Children's Hospital, University Medical Center Groningen, P.O. Box 30.001, 9700 RB Groningen, The Netherlands. Tel: +31503612800 ; Fax: +31 503611 4235; E-mail: r.m.f.berger@bkk.umcg.nl

Accepted for publication 1 May 2009 and the underrepresentation of associated as opposed to idiopathic pulmonary arterial hypertension, this data has provided evidence that new therapeutic strategies improve symptoms, exercise capacity, haemodynamics, and outcome in these patients. These trials have led not only to the current approval of seven different drugs for pulmonary arterial hypertension, but also to evidenced-based algorithms for treating patients with this rare disease.

\section{Congenital cardiac disease}

Congenital malformations of the heart occur in from 0.6 to $0.8 \%$ of all live newborns. It has been suggested that up to one quarter of these patients develop pulmonary arterial hypertension. ${ }^{4,5}$ Reliable data on such prevalence for patients with congenitally malformed hearts are lacking, but due to the continuously improving prognosis of children with such lesions, of whom up to nine-tenths are believed to survive into adulthood, the prevalence is increasing. Although absolute numbers of patients with congenital cardiac disease may be small compared to other cardiovascular diseases, when it is appreciated that the criterion for diagnosis of an orphan disease is a prevalence of fewer than 5 for 10.000 persons, congenital heart diseases certainly do not qualify.

Randomized controlled trials investigating the efficacy of treatment of patients with congenitally malformed hearts are extremely scarce. This may be explained by several factors, including the marked heterogeneity within patients having the congenital cardiac malformations, the inherent difficulties in 
performing randomized trials so as to evaluate the strategies for surgical treatment, the dispersion of patients over small centers, and the involvement of often young children. Irrespective of these reasons, the consequence is that evidence-based medicine hardly exists to guide the management of patients with congenital heart disease. During the last 50 years, an extensive body of expertise has been assembled, and empirical evidence has been gathered, initially by the pioneers in the field, with subsequently refinements from paediatric and congenital cardiologists and congenital cardiothoracic surgeons. Scientific evidence for many strategies, nonetheless, continues to be scarce. Management of patients with congenital heart disease is frequently driven by expert opinions.

\section{Pulmonary arterial hypertension associated with congenital cardiac disease}

So where does the situation described above leave the patients with "pulmonary arterial hypertension associated with congenital heart disease"? To start, the phrase is a rather confusing and misleading terminology. In the current classification for pulmonary hypertension, markedly different diseases are classified into one common group, namely pulmonary arterial hypertension, because of similarities in the histopathology and the course of the accompanying pulmonary vascular disease. These common features include the progressive nature of the vascular disease, and the presence of characteristic vascular lesions. ${ }^{2}$

In those with congenitally malformed hearts, it is recognized that these characteristic features of pulmonary arterial hypertension are virtually exclusively associated with the presence, or history, of a significant left-to-right shunt causing increased flow of blood to the lungs. This increased flow, with or without associated initial increase in pulmonary arterial pressure, is believed to induce specific injury to the pulmonary vasculature, thus leading to the characteristic vascular disease of pulmonary arterial hypertension in susceptible persons. The haemodynamic characteristics of the pulmonary-to-systemic shunt clearly affect the evolution of the vascular disease. According to the current classification, in patients with congenitally malformed hearts disease, it is only the pulmonary hypertension "associated with congenital systemic-to-pulmonary shunt" that is assigned to "class-I, pulmonary arterial hypertension". ${ }^{2}$

But, in patients with congenitally malformed hearts, the symptoms, exercise capacity, clinical course and prognosis all depend on the type of the cardiac defect, whether the shunt is pre- or posttricuspid, the state of repair, and the presence of associated cardiac or extracardiac lesions. This is also the case in patients with pulmonary arterial hypertension associated with systemic-to-pulmonary shunts. Fully to understand in the individual patient, therefore, the relation between coexisting pulmonary hypertension and a congenital cardiac malformation, it is necessary adequately to define the cardiac malformation from both the morphological and physiological points of view. ${ }^{6}$ Put another way, in patients with pulmonary arterial hypertension associated with "congenital systemic-topulmonary shunt", the pulmonary vascular disease and its characteristics are indeed similar to that in patients with other forms of pulmonary arterial hypertension, but the clinical course and prognosis for the patient are not necessarily comparable. This, for example, is illustrated by the far more favourable survival in patients with the Eisenmenger syndrome when compared to those with other forms of pulmonary arterial hypertension.

Another unique aspect of pulmonary arterial hypertension in patients with systemic-to-pulmomary shunts is the fact that, clinically, a reversible stage of the disease can be recognized, in which repair of the cardiac defect leads to regression of the pulmonary vascular disease, and even functional normalization of the pulmonary vasculature. It is obvious that the most optimal and long lasting treatment of patients with pulmonary arterial hypertension associated with congenital systemicto-pulmonary shunts is closure of the shunt before the pulmonary vascular disease has progressed to an advanced and irreversible stage. Unfortunately, the determination of the progression of the vascular disease, and the time point at which this reversible stage has progressed into an irreversible stage, is hazardous.' Although it has been part of the preoperative evaluation of children with congenitally malformed hearts since surgical correction started almost 50 years ago, even today no adequate haemodynamic criterions, or histological criterions for evaluation of lung biopsies, or indeed any other criterions, exist to define this "point of no return". Nor are there yet agreed features with which to assess the claimed potential of the new "antiproliferative" drugs to shift advanced pulmonary vascular disease towards more reversible disease. Currently, every centre treating patients with congenital heart diseases has its own approach to determine whether an individual patient in the grey area between reversible and advanced disease may still be a candidate for closure of the defect. Such evaluation has been part of our clinical practice for more than 40 years, yet data and consensus on this issue are extremely thin. Presentation of the data from such "borderline" patients to an audience of specialists in the field of congenital heart disease vouches for 
never-ending discussion and dispute, illustrating the lack of consensus and the need for appropriate data.

In patients with congenital systemic-to-pulmonary shunt in who pulmonary vascular disease has advanced, and the pulmonary vascular resistance equals or exceeds systemic vascular resistance, the original left-to-right shunt reverses into a right-to-left shunt, leading to cyanosis, the so-called Eisenmenger reaction. Eisenmenger's syndrome is not, however, restricted to the heart and pulmonary vasculature, but is a multi-organ disease associated with haematological and haemostatic abnormalities. During its course, patients may encounter thromboembolic complications, renal dysfunction, cerebrovascular accidents, develop gall stones, uricemia and gouty arthritis, and hypertrophic osteoarthropathy.

Supportive therapy is generally advocated as the mainstay of management of these patients. The aim is to reduce symptoms, and to treat or prevent the complications of cyanosis and haematological abnormalities, to prevent infection, and preserve the fluid balance so as to avoid dehydration. ${ }^{6}$ Venesections exacerbate iron deficiency, and increase rather than decrease the risk of stroke and, thus, seems best avoided. Advice concerning lifestyle includes the avoidance of pregnancy, strenuous exercise and competitive sports, and the need for annual immunization against influenza and pneumococcal infections. Further, it includes careful planning of non-cardiac surgery, which carries significant risks of death in this population. Although no evidence exists for this supportive therapy, there is general consensus regarding its value in these patients. In contrast, there is no consensus on the benefits of new medical "pulmonary arterial hypertension-therapies" in patients with Eisenmenger's syndrome. This is remarkable, since the only randomized placebo-controlled trial ever performed in patients with the Eisenmenger syndrome was on the use of bosentan, and showed its beneficial effects in the short term. ${ }^{8}$

In summary, it is clear that data derived from patients with pulmonary arterial hypertension cannot be simply extrapolated to patients with pulmonary arterial hypertension associated with systemicto-pulmonary shunts. The presence of a congenital systemic-to-pulmonary shunt in patients with pulmonary arterial hypertension demands specific considerations with regards to diagnosis and clinical course, haemodynamic evaluation, potential surgical treatment, and potential effects of vasodilatory drugs. At the same time, clinical data derived from patients with congenitally malformed hearts, which might provide guidance in such considerations, is extremely scarce.
Statements on the management of pulmonary hypertension associated with congenital heart disease

It is to this background, that Lopes and Rabinovitch are to be commended for their achievement in bringing together worldwide experts in the field of congenital cardiac disease and pulmonary arterial hypertension, and to provide a statement-document on the management of pulmonary arterial hypertension associated with congenital heart disease, published as an online-supplement of this journal. ${ }^{9}$ Members of the "pulmonary hypertension and congenital heart disease taskforce" of the Pulmonary Vascular Research Institute, all renowned experts in the field, combined their efforts in reviewing the literature and discussing their experiences and opinions on several aspects of pulmonary hypertension in congenital heart disease. Giving the lack of available evidence, as illustrated by a maximal level of evidence, as provided by the authors, scarcely exceeding level $\mathrm{B}$, recommendation IIb for each statement throughout the document, it inevitably represents expert opinions more than evidence-based guidelines.

This, however, in no way diminished the importance of this document, since valuable expert guidance in the often difficult management of patients with pulmonary arterial hypertension associated with congenital heart disease is scarce, and when available is a valuable tool in the education for those who are less expert in this complex field. Just as important is that it may serve to unify our thoughts on the approaches and management in this heterogeneous group of patients, and to identify the important gaps, where we lack the data and evidence required to make important therapeutic decisions.

Studies in patients with congenitally malformed hearts are often complicated by the heterogeneity of the cardiac defects. Hence, there are relatively small numbers of homogeneous patients. Those dealing with such patients should now follow the example of the investigators of pulmonary hypertension, who have succeeded in performing consecutive randomized controlled trials investigating new therapeutic strategies despite the relatively low numbers of patients associated with an orphan disease. In this way, they have accumulated data to provide a basis for evidencebased guidelines for those treating patients with pulmonary arterial hypertension. How was this accomplished? It was achieved by collaborating intensely in a strong global network, by performing multicentric trials, and by reaching generally accepted definitions, criterions, and objectives in a heterogeneous population. We should learn from them, and hopefully the initiative of Lopes and Rabinovitch will be but the first step in this direction. 


\section{References}

1. Peacock AJ, Murphy NF, McMurray JJ, Caballero L, Stewart S. An epidemiological study of pulmonary arterial hypertension. Eur Respir J 2007; 30: 104-109.

2. Simonneau G, Galiè N, Rubin LJ, Langleben D, Seeger W, Domenighetti G, Gibbs S, Lebrec D, Speich R, Beghetti M, Rich S, Fishman A. Clinical classification of pulmonary hypertension. J Am Coll Cardiol 2004; 43 (12 Suppl S): 5S-12S.

3. Galiè N, Manes A, Negro L, Palazzini M, Bacchi-Reggiani ML, Branzi A. A meta-analysis of randomized controlled trials in pulmonary arterial hypertension. Eur Heart J 2009; 30: 394-403.

4. Duffels MG, Engelfriet PM, Berger RMF, van Loon RL, Hoendermis E, Vriend JW, van der Velde ET, Bresser P, Mulder BJ. Pulmonary arterial hypertension in congenital heart disease: an epidemiologic perspective from a Dutch registry. Int J Cardiol 2007; 120: 198-204.

5. Engelfriet PM, Duffels MG, Möller T, Boersma E, Tijssen JG, Thaulow E, Gatzoulis MA, Mulder BJ. Pulmonary arterial hypertension in adults born with a heart septal defect: the Euro Heart Survey on adult congenital heart disease. Heart 2007; 93: 682-687.

6. van Albada ME, Berger RMF. Pulmonary arterial hypertension in congenital cardiac disease-the need for refinement of the Evian-Venice classification. Cardiol Young 2008; 18: $10-17$.

7. Berger RMF. Possibilities and impossibilities in the evaluation of pulmonary vascular disease in congenital heart defects. Eur Heart J 2000; 21: 17-27.

8. Galiè N, Beghetti M, Gatzoulis MA, Granton J, Berger RMF, Lauer A, Chiossi E, Landzberg M, Bosentan Randomized Trial of Endothelin Antagonist Therapy-5 (BREATHE-5) Investigators. Bosentan therapy in patients with Eisenmenger syndrome: a multicenter, double-blind, randomized, placebo-controlled study. Circulation 2006; 114: 48-54.

9. Lopes AA, Rabinovitch M. Statements on the management of pulmonary hypertension associated with congenital heart disease. Cardiol Young 2009; E-Supplement 1: 1-53. 